\title{
Spectral properties of the Dirichlet-to-Neumann operator for exterior Helmholtz problem and its applications to scattering theory
}

\author{
Lakshtanov E.L.* ${ }^{* \dagger}$
}

\begin{abstract}
We prove that the Dirichlet-to-Neumann operator (DtN) has no spectrum in the lower half of the complex plane. We find several application of this fact in scattering by obstacles with impedance boundary conditions. In particular, we find an upper bound for the gradient of the scattering amplitude and for the total cross section. We justify numerical approximations by providing bounds on difference between theoretical and approximated solutions without using any a priory unknown constants.
\end{abstract}

\section{Introduction}

In this article we discuss some spectral properties of the so called Dirichlet-toNeumann map which allows to determine many properties of the scattering amplitude for scattering by obstacles with impedance boundary conditions. We remind the reader that, up to now, we did not have any concrete information on the scattering properties for obstacles of arbitrary shape in case of intermediate values of the frequency. Besides, in numerical schemes (like Galerkin's scheme, for example) all inequalities controlling the difference between theoretical and constructed solutions include some, a priori, unknown

${ }^{*}$ Department of Mathematics, Aveiro University, Aveiro 3810, Portugal. This work was supported by Centre for Research on Optimization and Control (CEOC) from the "Fundação para a Ciência e a Tecnologia" (FCT), cofinanced by the European Community Fund FEDER/POCTI, and by the FCT research project PTDC/MAT/72840/2006.

†e-mail: lakshtanov@rambler.ru 
constant, which depends on the surface. Our results on the spectrum of DtN allow to exclude this dependence.

The article has the following structure. First, we prove absence of the spectrum in the lower halfspace for the operator DtN. Then, theorem 2 states an upper bound for the difference between theoretical and approximated solution. Theorem 3 lists upper bounds for total cross section, gradient of the scattering amplitude, field on the boundary and its normal derivative. And finally, theorem 4 is a note on the wave analogue of the Newton's minimal resistance problem, namely we present a lower bound for the transport cross section.

Consider a bounded body $\Omega \subset \mathbb{R}^{3}$ with smooth boundary $\partial \Omega$ and $k>$ 0 . The scattered field is given by the Helmholtz equation and a radiation condition

$$
\begin{gathered}
\Delta u(r)+k^{2} u(r)=0, \quad r \in \Omega^{\prime}=\mathbb{R}^{3} \backslash \Omega, \\
\int_{|r|=R}\left|\frac{\partial u(r)}{\partial|r|}-i k u(r)\right|^{2} d S=o(1), \quad R \rightarrow \infty,
\end{gathered}
$$

If we fix the quite smooth boundary condition on $\partial \Omega$,

$$
u(r)=u_{0}(r), \quad u_{0} \in W_{2}^{1 / 2}(\partial \Omega),
$$

then there exists a unique solution which satisfies all these conditions (eg [4]). Every function $u(r)$ which satisfies the mentioned conditions has asymptotic

$$
u(r)=\frac{e^{i k|r|}}{|r|} u_{\infty}(\theta)+o\left(\frac{1}{|r|}\right), \quad r \rightarrow \infty, \quad \theta=r /|r| \in S^{2},
$$

where the function $u_{\infty}(\theta)=u_{\infty}\left(\theta, k, u_{0}\right)$ is called the scattering amplitude and the quantity

$$
\sigma_{u_{0}}=\left\|u_{\infty}\right\|_{L_{2}\left(S^{2}\right)}^{2}=\int_{S^{2}}\left|u_{\infty}(\theta)\right|^{2} d \mu(\theta)
$$

is called the total cross section. $\mu$ is a square element of the unit sphere.

The operator $F$ which associates a boundary condition $u_{0} \in C(\partial \Omega)$ to the scattering amplitude $u_{\infty}$ is called the Far field operator. Its boundedness easily follows from the existence of the Dirichlet Green function [10, [11], therefore, it can be continued to a bounded operator $F: L_{2}(\partial \Omega, d S) \rightarrow$ $L_{2}\left(S^{2}, d \mu\right)$, where $d S$ is a standard square measure on $\partial \Omega$.

The operator $D t N: L_{2}(\partial \Omega) \rightarrow L_{2}(\partial \Omega)$ associates a function $u_{0}$ to the normal derivative of the corresponding field $u(r)$.

$$
\operatorname{DtN}\left(u_{0}\right)=\frac{d u}{d n}(r), \quad r \in \partial \Omega .
$$


Operator $D t N$ with domain $\left\{u_{0} \in W_{1 / 2}^{2}(\partial \Omega): D t N u_{0} \in L_{2}(\partial \Omega)\right\}$ is unbounded, pseudodifferential operator of order 1 with compact resolvent [16, Ch.7], [15],[18], [2, Th 3.11].

Theorem 1. The operator DtN has no spectrum in the lower half of $\mathbb{C}$.

Note that in the case where $\partial \Omega$ is a sphere this fact was known earlier (eg [17]).

Proof. Let us prove that for every function $u \in C^{2}\left(\mathbb{R}^{3} \backslash \Omega\right) \cap C^{1}\left(\overline{\mathbb{R}^{3} \backslash \Omega}\right)$ which satisfies (1),(2) we have

$$
\left\|\frac{\partial u}{\partial n}+(a+i b) u\right\|_{L_{2}(\partial \Omega)} \geq b\|u\|_{L_{2}(\partial \Omega)}
$$

where $a, b \in \mathbb{R}, \quad b>0$.

$$
\left\|\frac{\partial u}{\partial n}+(a+i b) u\right\|^{2}=\left\|\frac{\partial u}{\partial n}+a u\right\|^{2}+2 b \Im\left(\int_{\partial \Omega} \frac{\partial u}{\partial n} \bar{u} d S\right)+b^{2}\|u\|^{2} .
$$

The proof is finished by the well know fact (which follows from the Second Green's identity)

$$
\Im\left(\int_{\partial \Omega} \frac{\partial u}{\partial n} \bar{u} d S\right)=k\left\|u_{\infty}\right\|_{L_{2}\left(S^{2}\right)}^{2} \geq 0
$$

Note now that the the inverse to $[D t N+(a+i b)]$ is defined on a dense set in $L_{2}(\partial \Omega)$, since the boundary problem (11),(2) and

$$
(D t N+a+i b) u=f,
$$

are uniquely solvable for $f \in W_{2}^{1 / 2}(\partial \Omega)$ (eg. [4]). According to (51), it is bounded on this set and therefore can be continually extended to a bounded operator acting on $L_{2}(\partial \Omega)$. The theorem is proved.

\subsection{Justification of arbitrary numerical schemes with uniform constant}

Let the field $u^{\gamma}$ satisfy conditions (11),(2) and impedance boundary conditions of the form

$$
\left(\frac{\partial}{\partial n}+\gamma(r)\right) u^{\gamma} \equiv f(r), \quad r \in \partial \Omega, \quad f \in C(\partial \Omega) .
$$

where the function $f(r)$ is supposed to be known, the impedance function $\gamma(r) \in C(\partial \Omega)$ has positive imaginary part $\Im(\gamma(r)) \geq \gamma_{0}>0$ and $\gamma_{0}$ is 
a constant. The existence and uniqueness of the solution of (11),(2),(17) is proven, for example, in [1, 4].

Suppose that we have found a function $u^{\gamma, 1}$ that satisfies (11),(2) and almost satisfies (7) (it is not important how it was found, either by applying numerical schemes or using analytical approximations in case of small or large values of $k$ ):

$$
\left(\frac{\partial}{\partial n}+\gamma(r)\right) u^{\gamma, 1} \equiv f(r)+\alpha(r), \quad \alpha(r) \in L_{2}(\partial \Omega, d S) .
$$

In what follows, $\|\cdot\|=\|\cdot\|_{L_{2}(\partial \Omega)}$ and $\Gamma=\|\gamma\|_{C(\partial \Omega)}$.

Theorem 2. 1. We have an upper bound for the difference of fields:

$$
\left\|u^{\gamma}-u^{\gamma, 1}\right\| \leq \frac{1}{\gamma_{0}}\|\alpha\|
$$

2. There is an upper bound for the difference of normal derivatives:

$$
\left\|\frac{\partial}{\partial n} u^{\gamma}-\frac{\partial}{\partial n} u^{\gamma, 1}\right\| \leq\left(\frac{\Gamma}{\gamma_{0}}+1\right)\|\alpha\|
$$

3. And finally, there is an upper bound for the difference between total cross sections of theoretical and constructed waves:

$$
\left\|u_{\infty}^{\gamma}-u_{\infty}^{\gamma, 1}\right\|_{L_{2}\left(S^{2}\right)}^{2} \leq \frac{1}{k \gamma_{0}}\left(\frac{\Gamma}{\gamma_{0}}+1\right)\|\alpha\|^{2}
$$

The proof is given in part 4 .

\subsection{Scattering of a plane wave by obstacle with impedance boundary conditions}

Now we consider scattering of the incident field $e^{i k\left(r \cdot \theta_{0}\right)}$ formed by a plane wave with incident angle $\theta_{0} \in S^{2}$, by an obstacle $\Omega$. Let the field $u^{\gamma}$ satisfy conditions (11),(2) and impedance boundary conditions of the form

$$
\left.\mathcal{B}_{\gamma}\left(u^{\gamma}\right)\right|_{\partial \Omega} \equiv-\left.\mathcal{B}_{\gamma}\left(e^{i k\left(r \cdot \theta_{0}\right)}\right)\right|_{\partial \Omega}, \quad r=(x, y, z) \in \partial \Omega,
$$

where $\gamma(r) \in C(\partial \Omega)$ is a positive function such that $\Im(\gamma(r)) \geq \gamma_{0}>0, \gamma_{0}$ is a constant, and $\mathcal{B}_{\gamma}=(\partial / \partial n)+k \gamma(r)$. The operator $B_{i \gamma}$ appears as a stationary analogue of the $\frac{\partial}{\partial n}-\gamma(r) \frac{\partial}{\partial t}$ for the time-dependent wave equation. 
Theorem 3. Let $u^{\gamma}$ satisfy (11), (2), (12). Denote by $S$ the area of $\partial \Omega$. The following inequalities hold:

1. We have an upper bound for the total cross section:

$$
\sigma_{\gamma}=\left\|u_{\infty}^{\gamma}\right\|_{L_{2}\left(S^{2}\right)}^{2} \leq S \frac{(1+\Gamma)^{2}\left(\gamma_{0}+\Gamma\right)}{\gamma_{0}^{2}}
$$

2. We have an upper bound for the gradient of the scattering amplitude:

$$
\left|\nabla_{\theta} u_{\infty}^{\gamma}(\theta)\right| \leq \frac{\sqrt{S} k}{4 \pi} \frac{1+\Gamma}{\gamma_{0}}\left(k\left(\gamma_{0}+\Gamma\right)+k+1\right), \quad \theta \in S^{2} \subset \mathbb{R}^{3} .
$$

3. And finally there are bounds for the field and normal derivative of the field on the surface $\partial \Omega$.

$$
\left\|u^{\gamma}\right\| \leq \sqrt{S} \frac{1+\Gamma}{\gamma_{0}}
$$

4.

$$
\left\|\frac{\partial u^{\gamma}}{\partial n}\right\| \leq k \sqrt{S} \frac{(1+\Gamma)\left(\gamma_{0}+\Gamma\right)}{\gamma_{0}}
$$

In case of constant value of $\gamma(r)$ the first statement was proven in [7]. The $2^{\text {nd }}$ inequality is a consequence of the well known representation:

$$
u_{\infty}(\theta)=\frac{1}{4 \pi} \int_{\partial \Omega}\left(\frac{\partial u}{\partial n}+i k(n \cdot \theta) u\right) e^{-i k(\theta \cdot r)} d S(r) .
$$

We should note, that we used everywhere $|(n \cdot \theta)| \leq 1$, so these inequalities could be improved.

\section{Wave analogue of the Newton's problem of body minimal resistance}

In 1685 Newton published [13] the solution of his problem of minimal resistance. The body flies through a rarefied medium where particles do not mutually interact and have elastic collisions with the body's surface. Newton considered convex bodies of revolution embedded in a certain cylinder and having the same geometrical cross section $\sigma_{c l}$. He obtained an exact positive solution in this case. Recently, a body with zero resistance was constructed [14. It is interesting to study the problem of minimization of the resistance of a body in case of wave scattering. 
In the wave model of scattering by an obstacle, the observable corresponding to classical resistance, is the transport cross section (eg [6])

$$
R_{\gamma}\left(k, \theta_{0}, \Omega\right)=\int_{S^{2}}\left(1-\left(\theta \cdot \theta_{0}\right)\right)\left|f_{\gamma}(\theta)\right|^{2} d \theta .
$$

Note that by definition the resistance is normalized by the total cross section $R_{\gamma} / \sigma_{\gamma} \in[0,2]$ and clearly, in classical scattering, the border values of the segment $[0,2]$ can be attained. Of course, since the distribution of the scattered wave $f(\theta)$ is an analytical function, it can not equal a $\delta$-function and so $R_{\gamma}$ cannot be equal to zero. But, due to the quasiclassical effect, for large obstacles (or wave numbers) the infimum of the normalized $R_{\gamma} \backslash \sigma_{\gamma}$ could be 0 . To see this effect, one can fix $k$ and observe a sequence of prolate spheroids $(a=b=n, c=1 \backslash n, n \rightarrow \infty)$. By the results of [6] we have for every convex body

$$
\lim _{k \rightarrow \infty} \frac{R_{\infty}}{\sigma_{\infty}}=\frac{R_{c l}}{\sigma_{c l}}
$$

and this ratio could become arbitrary small in our sequence of spheroids.

Theorem 4. The following inequality holds

$$
R_{\gamma}>\frac{1}{2 \pi}\left(\frac{\sigma_{\gamma} \gamma_{0}}{k S}\right)^{2} \frac{1}{(1+\Gamma)^{2}\left(1+\Gamma+\gamma_{0}\right)^{2}}, \quad \Im \gamma>0 .
$$

Therefore, we can conclude that $R_{\gamma}$ has a positive infimum in the class of obstacles with fixed total cross section $\sigma_{\gamma}$ and uniformly bounded area $S$.

\section{Discussion of the results}

1. Inequality (13) (Theorem 2, part 1.) solves the question whether for certain $\gamma$ and $k>0$ there exists a sequence of smooth obstacles with uniformly bounded area such that $\sigma_{\gamma}$ tends to infinity.

Note that this fact is quite nontrivial, since plane waves transfer infinite energy and every part of it interacts with the obstacle, even if it is quite far from the obstacle.

2. There exist many numerical methods of obstacle reconstruction from scattering data. But (13) gives us the possibility to estimate the area of the obstacle immediately, since we measured the scattering amplitude for any body angle. See [3] for another approach.

3. Inequality (14) (Theorem 3 part 2) gives us the possibility to extrapolate values of the scattering amplitude in case it is only known on the some net.

4. Theorem 2 evidently tells us exactly when we have to stop our numerical scheme. 


\section{Proofs of results}

In what follows, $\widehat{\Gamma}=\|\Im(\gamma)\|_{C(\partial \Omega)}$.

Lemma 1. For every field $u \in C^{2}\left(\mathbb{R}^{3} \backslash \Omega\right) \cap C^{1}\left(\overline{\mathbb{R}^{3} \backslash \Omega}\right)$ which satisfies (11), (2)), (3)

$$
\left\|\frac{\partial u}{\partial n}+k \gamma(r) u\right\| \geq \gamma_{0} k\|u\| \text {. }
$$

\section{Proof.}

$$
\begin{gathered}
\left\|\frac{\partial u}{\partial n}+k \gamma(r) u\right\|=\left\|\frac{\partial u}{\partial n}+k \operatorname{Re}(\gamma) u+i k \widehat{\Gamma} u+i k(\Im(\gamma(r))-\widehat{\Gamma}) u\right\| \geq \\
\left\|\frac{\partial u}{\partial n}+k \operatorname{Re}(\gamma(r))+i k \widehat{\Gamma} u\right\|-\|k(\Im(\gamma(r))-\widehat{\Gamma}) u\| \geq \\
k \widehat{\Gamma}\|u\|-k\left\|\Im(\gamma(r))-\gamma_{0}\right\|_{C}\|u\| \geq k \gamma_{0}\|u\| .
\end{gathered}
$$

Here we used that $\left\|\Im(\gamma(r))-\gamma_{0}\right\|_{C} \leq \widehat{\Gamma}-\gamma_{0}$. The Lemma is proved.

Let us prove theorem 2, Using lemma 1, we get

$$
\gamma_{0}\left\|u^{\gamma}-u^{\gamma, 1}\right\| \leq\left\|\left(\frac{\partial}{\partial n}+\gamma(r)\right)\left(u^{\gamma}-u^{\gamma, 1}\right)\right\|=\|\alpha\|
$$

2. Using $(\partial / \partial n+\gamma(r))\left(u^{\gamma}-u^{\gamma, R}\right)=\alpha(r)$, we get

$$
\left\|\frac{\partial}{\partial n} u^{\gamma}-\frac{\partial}{\partial n} u^{\gamma, 1}\right\| \leq \Gamma\left\|u^{\gamma}-u^{\gamma, 1}\right\|+\|\alpha\| \leq\left(\frac{\Gamma}{\gamma_{0}}+1\right)\|\alpha\|
$$

3. Using (6)

$$
\begin{gathered}
\left\|u_{\infty}^{\gamma}-u^{\gamma, 1} \infty\right\|_{L_{2}\left(S^{2}\right)}^{2} \leq \frac{1}{k}\left\|u^{\gamma}-u^{\gamma, 1}\right\| \cdot\left\|\frac{\partial}{\partial n} u^{\gamma}-\frac{\partial}{\partial n} u^{\gamma, 1}\right\| \leq \\
\frac{1}{k} \frac{1}{\gamma_{0}}\|\alpha\|\left(\frac{\Gamma}{\gamma_{0}}+1\right)\|\alpha\|=\frac{1}{k \gamma_{0}}\left(\frac{\Gamma}{\gamma_{0}}+1\right)\|\alpha\|^{2}
\end{gathered}
$$

Theorem 2 is proven.

Now, let us prove theorem [3. Note that from (12), it follows that

$$
\begin{aligned}
\left\|\frac{\partial u^{\gamma}}{\partial n}+k \gamma(r) u^{\gamma}\right\| & =\left\|\frac{\partial e^{i k\left(r \cdot \theta_{0}\right)}}{\partial n}+k \gamma(r) e^{i k\left(r \cdot \theta_{0}\right)}\right\| \leq \\
& \leq\left\|\frac{\partial e^{i k\left(r \cdot \theta_{0}\right)}}{\partial n}\right\|+k \Gamma\left\|e^{i k\left(r \cdot \theta_{0}\right)}\right\| \leq \sqrt{S} k(1+\Gamma)
\end{aligned}
$$


Recall that $S=\operatorname{Area}(\partial \Omega)$. Hence, using (19), we obtain

$$
\gamma_{0}\left\|u^{\gamma}\right\| \leq \sqrt{S}(1+\Gamma)
$$

Also from (12), we have

$$
-\frac{\partial u^{\gamma}}{\partial n}=k \gamma(r) u^{\gamma}(r)+\frac{\partial e^{i k\left(r \cdot \theta_{0}\right)}}{\partial n}+k \gamma(r) e^{i k\left(r \cdot \theta_{0}\right)},
$$

therefore

$$
\begin{gathered}
\left\|\frac{\partial u^{\gamma}}{\partial n}\right\| \leq k \Gamma\left\|u^{\gamma} \mid+\right\| \frac{\partial e^{i k\left(r \cdot \theta_{0}\right)}}{\partial n}\|+k \Gamma\| e^{i k\left(r \cdot \theta_{0}\right)} \| \leq \\
k \Gamma\left\|u^{\gamma}\right\|+\sqrt{S} k(1+\Gamma) \leq 2 k \sqrt{S}(1+\gamma)
\end{gathered}
$$

Now from (20) and (21), we have

$$
\sigma_{\gamma} \leq \frac{1}{k}\left\|u^{\gamma}\right\|\left\|\frac{\partial u^{\gamma}}{\partial n}\right\| \leq \frac{1}{k}\left(\frac{\sqrt{S}(1+\Gamma)}{\gamma_{0}}\right)(2 k \sqrt{S}(1+\Gamma))=\frac{2 S(1+\Gamma)^{2}}{\gamma_{0}} .
$$

This ends the proof of the theorem 3 .

Now we prove theorem 4. From (17), we obtain the upper bound for the scattering amplitude for every angle:

$$
\begin{gathered}
|f(\theta)| \leq \frac{1}{4 \pi}\left(\left\|\frac{\partial u_{\gamma}}{\partial n}\right\|+k\left\|u_{\gamma}\right\|\right) \sqrt{S}=\frac{k S}{4 \pi \gamma_{0}}(1+\Gamma)\left(\gamma_{0}+\Gamma+1\right)=: M, \quad \theta \in S^{2} . \\
R_{\gamma}=\int_{0}^{\pi} \int_{0}^{2 \pi}(1-\cos \widetilde{\theta})|f(\widetilde{\theta}, \varphi)|^{2} d(-\cos \widetilde{\theta}) d \varphi \geq \\
\int_{\widetilde{\theta}: 1-\cos \widetilde{\theta}>\delta} \int_{0}^{2 \pi}(1-\cos \widetilde{\theta})|f(\widetilde{\theta}, \varphi)|^{2} d(-\cos \widetilde{\theta}) d \varphi
\end{gathered}
$$

where $1 \geq \delta \geq 0$ is an arbitrary number. Using (23) we obtain that the last expression is greater than

$$
\delta\left(\sigma_{\gamma}-\int_{\widetilde{\theta}: 1-\cos \widetilde{\theta}<\delta} \int_{0}^{2 \pi}|f(\widetilde{\theta}, \varphi)|^{2} d(-\cos \widetilde{\theta}) d \varphi\right) \geq \delta\left(\sigma_{\gamma}-2 \pi \delta M^{2}\right) .
$$

Choosing $\delta:=\frac{\sigma_{\gamma}}{4 \pi M^{2}}$ we obtain

$$
R_{\gamma} \geq \frac{\sigma_{\gamma}^{2}}{8 \pi M^{2}}=\frac{1}{2 \pi}\left(\frac{\sigma_{\gamma} \gamma_{0}}{k S}\right)^{2} \frac{1}{(1+\Gamma)^{2}\left(1+\Gamma+\gamma_{0}\right)^{2}}
$$

Theorem 4 is proven. 


\section{References}

[1] A.N.Tychonov, A.A.Samarsky, "Equations of Mathematical Physics Pergamon", Oxford, (1963).

[2] David L. Colton, Rainer Kress, Inverse Acoustic and Electromagnetic Scattering Theory, Series: Applied Mathematical Sciences , Vol. 93, 1998.

[3] David Colton, Michele Piana, Inequalities for inverse scattering problems in absorbing media, Inverse Problems, 17 (2001), 597-605.

[4] A.G.Ramm, "Scattering by Obstacles" (Dordrecht: Reidel), (1986)

[5] A. Majda, "High frequency Asymptotics for the Scattering matrix and the inverse problem of Acoustical scattering", Comm. pure and applied math. vol. XXIX, 261-291, (1976)

[6] A.I.Aleksenko, W. de Roeck, E.L.Lakshtanov, "Resistance of the Sphere to a Flow of Quantum Particles", J.Phys. A.Math.Gen, (39), pp. 4251-4255, 2005.

[7] A.Aleksenko, P. Cruz, E. Lakshtanov, "High-frequency limit of the transport cross section in scattering by an obstacle with impedance boundary conditions", 2008 J. Phys. A: Math. Theor. 41255203

[8] W. de Roeck, E.L.Lakshtanov, "Total cross section exceeds transport cross section for quantum scattering from hard bodies at low and high wave numbers", J.Math.Phys, 48, 2007.

[9] A. Majda, M.E.Taylor, "The asymptotic behavior of the diffractive peak in classical scattering", Comm. pure and applied math. vol. XXX, 639-669, (1977)

[10] S. Gutman, A.G. Ramm, Numerical implementation of the MRC method for obstacle scattering problems, J. Phys. A: Math. Gen. 35 (2002) 8065-8074

[11] S. Gutman, A.G. Ramm, Modified Rayleigh Conjecture Method and Its Applications, arXiv:math/0601298v1 [math.NA]

[12] Ramm. A.G., Calculation of the scattering amplitude for the wave scattering from small bodies of an arbitrary shape, Radiofisika, 12, (1969), 1185-1197. 43,7131. 
[13] I. Newton, Philosophiae naturalis principia mathematica, 1686.

[14] Alena Aleksenko, Alexander Plakhov, Bodies of zero resistance and bodies invisible in one direction, Nonlin 22(6), pp. 1247-1258, 2009.

[15] G. Uhlmann, Inverse boundary value problems and applications, Asterisque, 207 (1992), pp. 153-211.

[16] M.E. Taylor, Partial Differential Equations II: Qualitative Studies of Linear Equations. Springer-Verlag, New-York. 1996.

[17] J.-C. Nédélec, Acoustic and Electromagnetic Equations, Integral Representations for Harmonic Problems, Springer-Verlag, 2001.

[18] B. R. Vainberg and V. Grushin, Uniformly nonelliptic problems, Math. USSR-Sbornik, 2 N1 (1967), pp. 111-133. 